\title{
Peruana del bicentenario: promotora del emprendimiento en tiempos de crisis
}

\section{Peruvian woman in the bicentennial: Entrepreneurship promoter in times of crisis}

Jessica Aleida Mendoza Aranzamendi ${ }^{1 . a}$ 0000-0001-9780-3683

Yenny Marilú Pinto Villar ${ }^{1, b}$ 0000-0002-9813-1636

Mario César Gálvez Marquina ${ }^{1, \mathrm{c}} \underline{0000-0002-8948-5520}$

${ }^{1}$ Universidad Nacional Jorge Basadre Grohmann, Tacna, Perú.

aimendozaa@unjbg.edu.pe

bypintov@unjbg.edu.pe

cmariogalvez110@gmail.com

\author{
Recibido el: 01/10/2021
}

Aceptado el: 14/11/2021

\section{Resumen}

Aún no se define con claridad las competencias empresariales y acceso a los mercados para generar ingresos económicos por parte de las mujeres como promotoras del emprendimiento en tiempos de pandemia por COVID-19; por tales consideraciones, la presente investigación tiene por objetivo establecer las características de las citadas competencias de la mujer como emprendedora en el marco del Bicentenario del Perú, en época de crisis. La investigación se enmarca en la metodología de enfoque cualitativo; y, de diseño fenomenológico hermenéutico. Se entrevistó a 15 emprendedoras de distintos rubros; y los resultados evidencian que la mujer debe compartir un rol en el hogar mientras busca emprender para mejorar su economía y afrontar la crisis. Se concluye que la emprendedora presenta dificultades debido a sus limitaciones en materia de financiamiento, su rol social y el estereotipo de género. Emprender es su principal temor vinculado al descuido de su hogar. La identificación de oportunidades a través de un análisis del entorno no es una práctica planificada, por lo que se debería reforzar la cultura y las capacidades de planificación a mediano y largo plazo. Se han adaptado a la tecnología para manifestar sus emprendimientos y hacer valer sus habilidades creativas, conocimientos e innovación en productos y servicios, descubriendo una diversidad de actividades posibles para desarrollar en sus tiempos libres. Resulta imprescindible fortalecer la igualdad de género en el emprendimiento para superar el problema de desigualdad frente a los hombres.

Palabras clave: bicentenario, pandemia, COVID 19, crisis económica, igualdad de género, emprendedora.

\begin{abstract}
The entrepreneurial competencies and access to markets to generate economic income by women as promoters of entrepreneurship in times of COVID-19 pandemic are still not clearly defined; for such considerations, the present research aims to establish the characteristics of the aforementioned competencies of women as entrepreneurs in the framework of the Peruvian Bicentennial, in times of crisis. The research is framed within the methodology of qualitative approach; and, of hermeneutic phenomenological design. We interviewed 15 women entrepreneurs from different fields; and the results show that women must share a role in the household while they seek to undertake to improve their economy and face the crisis. It is concluded that women entrepreneurs have difficulties due to their limitations in terms of financing, their social role and gender stereotypes. Entrepreneurship is her main fear linked to the neglect of her home. The identification of opportunities through an analysis of the environment is not a planned practice, so the culture and capacities for medium-and long-term planning should be strengthened. They have adapted to technology to manifest their entrepreneurship and assert their creative skills, knowledge and innovation in products and services, discovering a diversity of possible activities to develop in their free time. It is essential to strengthen gender equality in entrepreneurship in order to overcome the problem of inequality in relation to men.
\end{abstract}

Key words: bicentennial, pandemic, COVID 19, economic crisis, gender equality, entrepreneurship. 


\section{Introducción}

La palabra emprendedor proviene del término francés entrepreneur que significa pionero (Surya \& Ati, 2022), fue introducido inicialmente por Richard Cantillo en 1755 y es la acción que busca crear un negocio con el que se espera generar ganancias (Ebabu, 2021). Es fundamental para que sectores económicos nuevos crezcan, se transformen y desarrollen (Beriso, 2021). Gracias al emprendimiento se crean empresas que generan autoempleo y es una importante modalidad para generar ingresos en economías en vías de desarrollo (Querejazu, 2020). Asimismo, esta actividad se considera una alternativa para enfrentar la crisis económica que viven muchas personas (Galindo et al., 2021). El emprendimiento se impulsa por factores externos como el contexto económico, político o las tecnologías; mientras que los factores internos son las motivaciones, la actitud, la aptitud intelectual y técnica y los atributos del carácter (Guananga et al., 2018).

En países de América Latina, entre el 18 y $30 \%$ de la población económicamente activa desarrolló actividades emprendedoras durante la década pasada, a diferencia de Estados Unidos que alcanzó el $10 \%$ y países de la Unión Europea que alcanzó el $6 \%$ (Querejazu, 2020). La emprendedora latinoamericana tiene una edad entre 35 y 45 años, aspecto que les favorece por el nivel de experiencia, información, y capacidades para crear empresa, pero que se relaciona con su perfil de madre y mujer trabajadora con personas a cargo, que limita el lograr mejores desempeños y dedicación a sus proyectos de negocio (Ruedas, J. \& Ruiz, R.,2019). Para vender sus productos utilizan estrategias de penetración y permanencia en el mercado, identificar clientes y proveedores, y retener clientes (Arteaga et al., 2020).

El emprendimiento sostiene a la economía mundial e históricamente se ha vinculado a los hombres (Santander, 2019), pero, en recientes investigaciones se evidencia que la participación de las mujeres se ha incrementado notablemente, especialmente en Latinoamérica, cuyos índices de emprendimiento son elevados en hombres y mujeres (Zegarra et al., 2020). Este ascenso se debe, entre otros factores, a la escasa oportunidad de acceder a puestos laborales para las mujeres en comparación a los hombres, por ello, en el caso de países como Ecuador, el emprendimiento se origina por necesidad y no por oportunidad (Delgado et al., 2020; Uzcategui et al., 2019; Zambrano \& Beltrán, 2017).

También se ha identificado que la necesidad no es un factor condicionante para el desarrollo de un negocio apresurado. El planeamiento, y no la urgencia, hace que se evite lanzar al mercado productos precipitadamente, como ocurre con las emprendedoras chilenas, quienes, generalmente, preparan el emprendimiento por más de tres meses, a pesar de que la mitad de emprendedoras está motivada por necesidades económicas (Barbagelata, 2019), asimismo, los servicios es el sector en el que más incursionan, seguido del sector comercio (Zegarra et al., 2020).

En Colombia, se observa que de cada 10 emprendedores 7 son mujeres y las que emprenden microempresas va incrementándose anualmente; así, en el 2020 las mujeres habían emprendido el $43 \%$ de empresas del mercado (Cámara de Comercio de Cali, 2021).

En el Perú, el $63 \%$ de emprendedoras tiene estudios técnicos completos, el $44 \%$ asume riesgos, el $68 \%$ está dispuesta a aprender de sus errores, el $65 \%$ tiene otros ingresos estables, el $82 \%$ está dispuesta a aprender, el $50 \%$ considera que la edad es importante para emprender y es más fácil emprender para las jóvenes (Vásquez, 2018).

Antes de la pandemia por COVID-19, el $45 \%$ de los emprendimientos lo hacían las mujeres; luego de iniciarse la pandemia, se incrementó a un 68 $\%$. El emprendimiento por necesidad tiene mayor índice que el de oportunidad. Para el $38 \%$ de emprendedoras el emprendimiento es la principal fuente de ingresos, para el $22 \%$ es una fuente temporal y para $33 \%$ es una fuente importante (DATUM, 2020).

En la ciudad de Tacna el $62 \%$ de emprendimiento es de mujeres como se observa en las localidades de de Quilahuani, Candarave y Sitajara (Wong et al., 2021). Su éxito no solo se sustenta en habilidades, talento o destrezas adquiridas, sino también en su visión, responsabilidad y proactividad junto a grandes dosis de resiliencia (Uzcategui et al., 2017.

Sin embargo, aún existen grandes desafíos que deben afrontar las emprendedoras; principalmente, por su encasillamiento al rol doméstico como parte de la construcción cultural. Ello las restringe, encasillándolas únicamente a funciones 
familiares (Tejeiro et al., 2021; Sabater, 2018), es decir, que, además de ser un sujeto activo en el proceso productivo, deben asumir con las responsabilidades del hogar que aún son consideradas tareas femeninas (Ordoñez et al., 2021). También tienen escasa financiación y, en algunos países, no acceden a créditos fácilmente por la poca credibilidad que se les otorga (Encina \& López, 2021), por lo que, casi siempre, acuden a sus ahorros (Velásquez \& Portocarrero, 2019). Por ello, es necesario fomentar el emprendimiento femenino con capacitaciones para que desarrollen competencias empresariales y acceso a los mercados (Alene, 2020).

El objetivo del presente estudio es establecer las características de las competencias de la mujer emprendedora en el Bicentenario de la Independencia de la República del Perú y cómo se encuentra establecido su rol en condición de empresaria en la ciudad de Tacna (Perú), ciudad que permaneció en poder del vecino país de Chile durante 50 años (1879-1929) como consecuencia de la guerra del Pacífico, donde la mujer tacneña ha destacado por su valentía. Ante los cambios sociales emergentes la mujer continúa afrontando nuevos desafíos para mejorar su economía con la inserción al ciclo productivo y el emprender en igualdad de género.

\section{Método}

La presente investigación, por sus características, se enmarca dentro del enfoque cualitativo, el diseño fue fenomenológico hermenéutico, con el que permitió recopilar e interpretar las experiencias de las mujeres en su rol de emprendedoras del Bicentenario. Para recolectar los datos se aplicó la entrevista y, como instrumento, un cuestionario no estructurado de preguntas abiertas, adaptando las competencias de emprendimiento desarrolladas por Alcazar y Villasana (2015), que fue reestructurado de acuerdo con cada entrevista y los datos de recolección, de tal manera que cada mujer emprendedora enriqueció la información con su experiencia vivida. La muestra estuvo determinada por el aporte de datos novedosos que brindaron las participantes, pues el interés es la profundidad y no la extensión (Hernández \& Mendoza, 2018); así, se procedió a entrevistar a 15 mujeres emprendedoras. Los datos fueron analizados siguiendo las fases propuestas por Fuster (2019), las cuales son: clarificación de presupuestos, recojo de experiencias de vida, reflexión sobre la experiencia vivida (etapa estructural) y escribirreflexionar acerca de las experiencias recopiladas.

\section{Resultados y discusión}

\section{Rol social de la mujer emprendedora y la igualdad de género}

Desde la Independencia del Perú, el rol exclusivo de la mujer como ama de casa y madre de familia hasta la actualidad, ha tenido importantes cambios; sin embargo, aún falta mucho para que estos se consoliden en una estructura social de equidad, en el que, no solo se le permita a la mujer desarrollar actividades que antes eran consideradas exclusivamente masculinas, sino que, fundamentalmente, los hombres desarrollen equitativamente las acciones que están encasilladas como propias de la mujer, sobre todo, si es que la mujer quiere emprender, pues, en los datos recopilados se ha podido evidenciar que el rol emprendedor de la mujer del bicentenario debe sumarse a las tareas que se le han asignado socialmente, tal como manifestó la Emprendedora 5, quien dijo: "Ha aumentado la autosuficiencia de la mujer, pero es un reto no descuidar el hogar sacrificando a la familia, especialmente, cuando los hijos son pequeños"; la Emprendedora 12 dijo: "No es que emprendamos y nos olvidemos de la casa, tenemos que sumarla, es decir, además de emprender nos recargamos de las tareas en el hogar, cosa que los hombres no hacen" y la Emprendedora 13 dijo: "Es difícil ser mamá emprendedora, el hombre va a trabajar y no se le reclama el cuidado de los hijos como a nosotras. Para ellos, el trabajo está primero y, para nosotras, la casa es lo primero".

En la última década se ha buscado impulsar la igualdad de género en el marco empresarial, intentado comprender la diferencia entre el emprendimiento femenino y masculino (Ordoñez et al., 2021); sin embargo, en términos de cultura y roles de género, en la mayoría de las sociedades, la responsabilidad de cuidar a los niños, ancianos o enfermos recae en las mujeres, mientras que a los hombres se les suele asignar el rol de proveedor.(Chávez et al., 2021) lo cual es aceptado por las mujeres en un gran porcentaje, siendo que en el Perú, el $60 \%$ de mujeres considera que su única responsabilidad es educar a sus hijos (Morrison, 2021). 
A pesar de las limitaciones sociales, no se han aplacado las ganas de superarse y emprender en igualdad de condiciones que los hombres, las mismas que manifiestan que se está mejorando, tal como indica la Emprendedora 08: "La mujer ha evolucionado, hoy podemos estudiar y trabajar para solventar los gastos familiares, eso nos motiva diariamente a poder superarnos en un entorno donde necesitamos trabajar" y la Emprendedora 09 dijo: "Nos inculcaron que los hombres eran responsables del sustento del hogar y de tomar las decisiones; hoy se ha roto el paradigma, ahora todo es compartido, la mujer apoya al igual que el hombre en el sustento familiar".

Aunque también, algunas entrevistadas, ven con escepticismo un cambio de la estructura social cuando en las familias peruanas no hay variaciones en la forma de educar, como la Emprendedora 15, quien dijo: "Los cambios son muy dificiles para llegar a una real igualdad es casi imposible, más aún porque las mismas mujeres incentivamos en los hogares la diferencia de roles entre hombres y mujeres al criar a nuestros hijos".

Por lo tanto, el rol social de la mujer del Bicentenario, comprende también surgir del encasillamiento social de su género, generando en el proceso de emprendimiento el deseo de superación, viendo en ello una oportunidad y un reto para poner de manifiesto su capacidad de progresar y ser el sustento económico de su familia; no obstante, resulta necesario fortalecer en los hogares la igualdad de género, la misma que debe gestarse en el seno familiar si se quiere lograr cambios en nuestras sociedad.

\section{Recuperarse y aprender del fracaso}

Algunas causas de los fracasos empresariales están al interior de la organización (Sopow, 2020), como la falta de planes de negocios, de formación y habilidades empresariales y, sobre todo, la falta de financiamiento y conocimiento para comercializar (Cichiello et al., 2021), tal como se halló en las entrevistas, así contamos con lo manifestado por la Emprendedora 11: "Mi primer negocio fue vender uniformes corporativos. Fracasó porque no tuve financiamiento para producir y vender continuamente, tenía que esperar que un cliente me pague para poder buscar otro, de lo contrario no tenía dinero para la nueva producción" o la Emprendedora 12 que dijo: "Emprendí con una empresa de comunicación corporativa, pero no supe cómo promocionarla, entonces no llegué a tener los clientes que me permitieran mantener el negocio".

Otro de los motivos principales que resaltan la mayoría de las entrevistadas, fue manifestar que sus primeros fracasos se relacionaron con el rol familiar, pues el emprendimiento se vio limitado por los quehaceres del hogar que impedían cerrar tratos comerciales. Al respecto, la Emprendedora 13 dijo: "Vendía ropa, pero era difícil salir con mi hijo recién nacido, no tenía el apoyo de su papá y no podía colocar la mercadería. Cuando la clienta venía tenía que descontarle el taxi. Finalmente tuve que dejar de vender" y la Emprendedora 14, explicó: "Tenía que bajar los productos de las zonas altoandinas y no había quien se encargue de las cosas de la casa, así que decidí ya no seguir llevando productos del campo a la ciudad”.

Asimismo, las emprendedoras manifestaron que al iniciar el emprendimiento sentían inseguridad por temor al fracaso, sin embargo, no fue un obstáculo para continuar, tal como lo describe Serida et al. (2018) quienes realizaron un estudio en 54 países en cinco continentes, concluyendo que la población de Latinoamérica y el Caribe sobresale en las capacidades necesarias para emprender $(58,8 \%)$, por poseer un bajo miedo al fracaso $(30,5 \%)$ y por contar con una alta intención a emprender $(31,3$ $\%)$. Estos resultados se reflejan en lo explicado por la Emprendedora 07: "Al principio tuve temor porque era algo nuevo ya que solo era un pasatiempo. Mi temor era fracasar, pero luego me di cuenta que mi producto tenía aceptación" y la Emprendedora 05: "Perseverancia, no es fácil al inicio, nadie te conoce, todos dudan en probar tu producto, ganarse al público y mantenerlo es un reto".

A pesar de ello, los fracasos iniciales trajeron importantes enseñanzas, especialmente porque aprendieron lo que no se debe hacer y cómo evitarlo en su nuevo emprendimiento, tal como mencionó la Emprendedora 12: "Como antes no podía salir a vender por mi hijo, me organicé con mi mamá en horarios en los que ella sí podía hacerlo y así podía llevar la mercadería" y la Emprendedora 13: "Los bancos no me prestaban, a pesar de que podía demostrar facturación, asi que me asocié con alguien que financia los diseños, las ganancias se reducen, pero puedo vender más". 
Se puede afirmar que los fracasos de las mujeres emprendedoras del Bicentenario, en su gran mayoría, se deben a las limitaciones y restricciones producto de su rol social que aún consideran están obligadas a ejercer solas, estereotipo que ha permanecido durante mucho tiempo y que forma parte de la historia. Afortunadamente, estos patrones sociales vienen desapareciendo, pero no al ritmo que la sociedad lo requiere, por lo que la mujer debe adaptarse a las necesidades del negocio emprendido sin descuidar sus labores del hogar. Contrariamente, si estas labores fueran compartidas con los hombres, se permitiría el establecimiento de negocios con mayor posibilidad de mantenerse en el mercado, lo cual beneficiaría a toda la familia a largo plazo.

La presente investigación pudo observar también que al inicio del emprendimiento hubo temor e inseguridad por parte de las participantes, pero su deseo de superación y en otros casos el descubrir una oportunidad en base a las actividades de pasatiempo, conocidas también como "hobby" permitiéndoles atenuar y superar dichos temores.

\section{Tolerancia al riesgo}

El riesgo es un escenario de incertidumbre en el negocio, accionando complicaciones por el inadecuado manejo administrativo, por lo que debe mejorar la gestión (Yopetech, 2021), por ello, es necesario que las emprendedoras analicen y verifiquen los riesgos más importantes que enfrentaron y evitar que el peligro las afecte en la rentabilidad de su negocio (Lin et al., 2021), como ha ocurrido con varias de las entrevistadas, quienes además de comprender los motivos que las llevaron a cerrar sus primeras actividades de emprendimiento, se han propuesto resolver buscando soluciones para evitar nuevos fracasos. Como es el caso de la Emprendedora 02 que mencionó: "Tuve que ahorrar para emprender. Recién hace dos meses puedo percibir ingresos propios, el riesgo lo he afrontado con mucha fe, innovo permanentemente y busco nuevos mercados...ello implica sacrificar tiempo con mi pareja pero tengo su apoyo", la Emprendedora 13 dijo: "Para que esta vez no cierre el negocio planifico bien mis horarios junto a la persona que me ayuda con mi hijo" y la Emprendedora 14 mencionó: "Ahora contacto con un intermediario, él es quien se lleva todo para vender, claro que no gano igual, pero, al menos puedo mantener la venta".
Podemos identificar que la principal incertidumbre de la mujer emprendedora es manejar su rol emprendedor, su rol familiar o su vida personal, y para poder superar este riesgo, muchas veces necesita del apoyo que le pueda brindar su entorno. El reconocer sus riesgos, producto de sus anteriores fracasos, les permite trazar nuevos planes de acción para obtener nuevas propuestas de emprendimiento, por lo que se puede decir que su tolerancia al riesgo está supeditado al aprendizaje generada por una experiencia previa.

\section{Identificación de oportunidades}

\section{Análisis del entorno}

Para identificar oportunidades es importante analizar el entorno. Las emprendedoras que observen adecuadamente el entorno y se planteen escenarios para fortalecer el negocio y prepararse para afrontar la crisis, pueden transformar las circunstancias oportunamente para potenciar su crecimiento (Marín, 2020), asimismo, para determinar oportunidades de su negocio, las emprendedoras se basaron en la demanda a través de las redes sociales, como refiere la Emprendedora 01: "Me di cuenta que en Facebook muchas mamás hacían pedidos de ropa de niños y también pedian ropas de mujer" y la Emprendedora 02: "Vi por Facebook que mucha gente pedía dulces en tiempo de cuarentena y pensé que sería una oportunidad".

La mayoría de emprendedoras gestó su negocio en época de pandemia, donde surgieron oportunidades de mercado debido al cambio en los hábitos de compra que ayudaron a impulsar sus emprendimientos, como lo precisa la Emprendedora 10: "Antes de la pandemia comencé con la venta de envases ecológicos, sin embargo, ahora hay una mayor demanda. También empecé a vender artículos para el hogar, con la pandemia la gente pasa más tiempo haciendo actividades en casa".

También consideraron oportunidades por casualidad. Inicialmente sus productos fueron creados como un pasatiempo que llamó la atención de familiares y amigos quienes fueron sus primeros clientes que, a su vez, la recomendaron, como lo menciona la Emprendedora 03: "Fue un hobby que se convirtió en negocio. Le vendo principiante a mujeres. Hago prendas personalizadas a mano y decoraciones para el hogar con la técnica de 
crochet" y la Emprendedora 07: "Lo hice por pasión. No pensé en el entorno. Solo emprendí con lo que más me gusta".

Las emprendedoras con negocios antes de la pandemia por COVID-19, tuvieron que identificar nuevas oportunidades para impulsar sus emprendimientos, como lo indica la Emprendedora 06: "Siempre quise un negocio que cuide el ambiente. Me di cuenta que en la cuarentena las mujeres estaban estresadas y buscaban fuentes de ingresos, así que comenzamos a capacitarlas para que emprendan negocios de cosmética natural".

Algunas emprendedoras reconocen que su emprendimiento no guarda relación directa con su profesión u oficio, pero los previos conocimientos adquiridos les ha servido de soporte para conducir sus negocios, como lo mencionó la Emprendedora 02: "Aplico técnicas de venta aprendidas en mi carrera de administración, pero mi emprendimiento es en repostería" y la Emprendedora 09: "Estudié administración y emprendi en el rubro textil, mis conocimientos en ventas y administración me ayudaron, pero no fue suficiente, tuve que capacitarme en textilería".

Se puede afirmar que las emprendedoras, si bien no manifiestan seguir un proceso sistemático para analizar su entorno incluso limitado, se han valido de fuentes de información como las redes sociales para identificar oportunidades de mercado. En otros casos, como consecuencia de la pandemia por COVID-19, que motivó al cambio de conducta de los consumidores, se generó una situación de amenaza para algunos negocios, pero para otros ha sido una oportunidad el cual ha sido aprovechado a través de la rápida innovación y adaptación.

También se observa que no todos los emprendimientos que se han gestado durante la crisis de pandemia por COVID-19, obedecen a una necesidad previamente identificada producto del análisis del entorno, todo lo contrario, se gestaron por una situación fortuita generada por un pasatiempo que al ser publicada en sus redes sociales tuvo aceptación entre su entorno cercano $\mathrm{y}$ luego fue trascendiendo y abriéndose a un mercado no atendido.

Por otro lado, se puede también evidenciar que los emprendimientos no siempre están relacionados con la experiencia o formación profesional de las emprendedoras, sino en aprovechar las oportunidades que se les presenta, lo cual implica fortalecer conocimientos que les permitan sacar la mayor ventaja posible.

\section{Iniciativa de emprender}

Otro aspecto importante es la iniciativa o espíritu emprendedor, que es una actitud general de mucha utilidad en la vida cotidiana o el desarrollo de cualquier actividad y está generando el autoempleo ante el elevado desempleo a raíz de los recientes cambios sociales y económicos (Salinas et al., 2013). Las razones que motivan el emprendimiento femenino en Latinoamérica es el aprovechamiento de oportunidades de negocio y necesidades de generar ingresos económicos, en la mayoría de los casos ante la pérdida de un puesto de trabajo.

En las emprendedoras entrevistadas, la iniciativa de emprender, se vio influenciada por la pérdida de empleos generada por la crisis económica a causa de la pandemia por COVID-19, así como por el cambio de prioridades en el consumo, lo que hizo que sus anteriores emprendimientos ya no tuvieran la misma demanda, así lo refirió la Emprendedora 01: "La pandemia y la cuarentena generó un problema económico y necesitábamos más ingresos", la Emprendedora 07 dijo: "Trabajaba en un hotel que cerró por la pandemia. Para distraerme en la cuarentena empecé a tejer. Al quedarme sin ingresos y viendo que a mis amistades les gustaba lo que tejía, poco a poco, empecé a vender".

La creencia en la autoeficacia aumenta la percepción de viabilidad en las mujeres $\mathrm{y}$, finalmente, influye en la intención y el resultado emprendedor (Molina et al., 2021), es decir, que, si se tiene conocimiento de sus cualidades y actitudes, ayudará al logro de sus metas de emprendimiento. Al respecto, la Emprendedora 03 mencionó: "Ser puntual, aprender a conocer el negocio para brindar productos de calidad, honestidad y responsabilidad, es lo que nos ha diferenciado del resto de competidores", la Emprendedora 07 dijo:

"La repostería requiere arte y dedicación y creo tenerlo, siempre me gusta hacer bien las cosas y que me identifiquen por algo" y la Emprendedora 08 manifestó: "Hacer algo que me gusta me hace superarme diariamente y se refleja en mis productos; he despertado habilidades creativas en pandemia y ahora busco crear productos con materiales reciclados. Me capacito constantemente para mejorar mi técnica de tejido". 
Por otro lado, respecto a los factores que impulsaron la iniciativa de emprender fue la pandemia por COVID-19, en los casos más recientes de emprendimiento, y la necesidad de generar un autoempleo, dada las circunstancias personales o profesionales que limitaban poder trabajar de forma dependiente en alguna empresa, lo cual coinciden con las investigaciones de diversos autores (Delgado et al., 2020; Uzcategui et al., 2019, Zambrano \& Beltrán, 2017).

Igualmente, las entrevistas permitieron establecer que las mujeres emprendedoras del Bicentenario presentan actitudes comunes, tales como el carisma, el trato amable con sus semejantes; y aptitudes logradas mediante la capacitación personal, así como cualidades de liderazgo y gestión en equipos de trabajo.

\section{Comunicación efectiva}

Otro elemento para identificar oportunidades es comunicar los emprendimientos de forma efectiva, más ahora que la nueva tecnología está teniendo un importante impacto en la comunicación de las empresas hacia sus clientes que generan valor preponderante en cuanto a rapidez, clara y óptima en transmitir mensajes. Para los clientes la tecnología representa un entretenimiento y un sentimiento de cercanía hacia la marca (Mele et al., 2021). Las redes sociales como Facebook, Tuenti, Twitter y Linkedin entre otros, cuentan cada vez con más seguidores, siendo un mecanismo para dar a conocer y posicionar marcas con pocos recursos y es un canal perfecto para las PYMES. El marketing boca a boca también está relacionado con el éxito del producto, es un medio en el cual un individuo comunica a otro, no las cualidades del producto, no con una intención comercial, sino dando a conocer su percepción personal de la calidad, generando confianza en el producto (Sun et al., 2021), asi lo manifiestan las Emprendedora 06: "Doy a conocer mi emprendimiento a través de Facebook y por referencias". Y la Emprendedora 08: "Uso redes sociales, correos electrónicos, WhatsApp"

Las emprendedoras se han adaptado con rapidez a las tendencias tecnológicas dada la actual coyuntura (pandemia por COVID-19); las redes sociales son los aliados a través del cual promocionan sus emprendimientos, siendo Facebook la más destacada por su frecuente y fácil acceso, seguida de la mensajería instantánea WhatsApp. Otro medio importante son referencias o publicidad boca a boca.

\section{Implementación de soluciones innovadoras}

\section{Innovación}

Respecto a la innovación para implementar soluciones innovadoras, a los emprendedores se les exige, habitualmente, que para iniciar su proyecto cuenten con una idea diferente, sorprendente y única. Sin embargo, la historia de los éxitos empresariales demuestra que una empresa puede triunfar sin disponer de una idea única de negocio (Medina, 2013). Las innovaciones puestas en marcha por las emprendedoras entrevistadas se basan en las necesidades o detalles que aprecian y valoran sus clientes, como lo contó la Emprendedoras 02: "El empaque es decorativo, a parte le incluyo una tarjeta con mis datos" y la Emprendedora 03 dijo: "Siempre le doy un plus, un bocadito de cortesía y una tarjetita con pensamientos animadores".

Es así, que los casos de emprendimiento de las entrevistadas no fueron ideas totalmente nuevas, sino con pequeños cambios que difieran de los productos existentes que han permitido su incorporación en función a la experiencia de ir conociendo a sus clientes y que ha sido valorados por los mismos, marcando la diferencia respecto al resto de su competencia.

\section{Planeación}

Sobre la Planeación de estas soluciones, las MIPYMES peruanas suelen enfocarse en el día a día de sus operaciones dejando de lado importantes espacios de planificación que permiten identificar qué han logrado y plantear objetivos en el corto, mediano y largo plazo (Global Reporting Initiative, 2021), así, la Emprendedora 01 manifestó: "Me he puesto metas, a largo plazo espero colocar un pequeño café y por ahora seguir manteniendo mis clientes" y la Emprendedora 10 dijo: "Al principio fue difícil, me enfoqué en vender, luego me di cuenta que tenía que analizar mejor mi mercado, investigar muy bien para poder tomar la decisión correcta del qué y cuándo vender".

Se extrae de estas experiencias que el proceso de planificación es una práctica aún endeble. En las situaciones donde el emprendimiento fue una situación prevista, lo que hicieron las emprendedoras 
fue definir a quiénes vender su producto, en algunos casos estableciendo metas de ventas, en otros, definiendo presupuestos $\mathrm{y}$, finalmente, en algunos teniendo una visión a largo plazo.

\section{Obtener recursos}

El Ministerio de la Mujer y Poblaciones Vulnerables, mediante la resolución ministerial 159-2021.MIMP (2021) menciona que desde la gesta de la independencia hasta la actualidad la mujer ha sobresalido en el ámbito político, social y cultural. Actualmente en el sector de los negocios, la mujer emprendedora inicia regularmente su negocio con el apoyo de terceros, ello ha implicado ejercer cualidades de liderazgo en sus grupos de trabajo. El liderazgo transformacional destaca en la mujer emprendedora desarrollar una labor en equipo que genere beneficios para los colaboradores como, por ejemplo, la confianza y la estimulación de participación (Macedo \& González, 2017), tal como lo corroboró la Emprendedora 03: "Mi equipo de trabajo es el personal de confección y corte, trabajamos por metas", la Emprendedora 09: "Mi personal a cargo son los que me ayudan en el manejo de las redes sociales" y la Emprendedora 10: "Mis hijos son mi equipo de trabajo, colaboran dándome ideas de diseño y me ayudan con los empaques".

En Latinoamérica las mujeres emprendedoras cumplen una función muy importante en el desarrollo económico impulsando el crecimiento y generando empleos en el $40 \%$ de la población más pobre. Pero, enfrentan muchas dificultades para financiar, mantener y hacer crecer un negocio, entre ellas un acceso limitado al capital y la tecnología, la falta de redes de mercadeo, recursos de conocimientos, obstáculos legales y normativos (Banco Mundial, 2017).

\section{Acceso al financiamiento}

El acceso al financiamiento muchas veces se convierte en una limitante para el desafío de emprender debido a la poca credibilidad que les otorgan las entidades financieras por el tamaño de su negocio, lo que restringe el acceso a un crédito en el sistema financiero. El emprendimiento se convierte en un riesgo financiero y personal (Encinas et al., 2021), lo cual, se ha podido comprobar según lo manifestado por la Emprendedora 05: "Tuve que usar mis ahorros y pedir préstamos para comprar máquinas, al principio fue difícil, los banco no me querían prestar el dinero, eso me desanimaba, pero me fui ganando la confianza por la puntualidad en mis pagos", la Emprendedora 06 dijo: "Trabajé en la administración pública y pude ahorrar para emprender" y la Emprendedora 07 dijo: "Comencé con capital propio generado por mis ahorros de mis anteriores trabajos".

Se ha puesto de manifiesto que la mujer emprendedora ha tenido dificultades para financiar su emprendimiento por un limitado acceso al capital de trabajo. Todas empezaron con sus propios ahorros producto de negocios y trabajos anteriores.

\section{Habilidades en la negociación}

El desarrollo de las habilidades de negociación directamente con los proveedores de mercadería no ha sido evidenciado en las emprendedoras, pues prefieren buscar nuevos abastecedores para obtener mercadería e insumos de calidad a costos reducidos, que negociar con los que ya tienen, lo cual sería una dificultad para la supervivencia y éxito del emprendimiento (Huamán, 2018). Al respecto, la Emprendedora 02 mencionó: "Tengo dos proveedores para insumos principales $y$ materiales de repostería, y no tengo proveedores para insumos de alta rotación, la forma de pago contra entrega por aplicativo y asumo el costo del envio", la Emprendedora 03 dijo: "Siempre busco relación calidad-precio. Me gusta que mis insumos sean de buena calidad, pero siempre busco donde me cuesten menos, no tengo proveedores especificos" y la Emprendedora 04 manifestó: "En cuanto al precio, negocio con mi proveedor, a más productos pedidos mayor descuento".

\section{Pensamiento analítico}

El pensamiento analítico suministra importantes aportes a las actividades del emprendimiento. Roth et al. (2011) explican que está relacionado con el proceso emprendedor, ello abarca la disposición a cambiar los valores de apertura (la autodirección, la estimulación y el logro), tal como la Emprendedora 07 indicó: "Cuando decidi emprender tuve que mejorar mi técnica porque mis clientes empezaron a mandar diseños que eran un reto, para ello, me seguí capacitando por internet", la Emprendedora 08 dijo: "Como administradora de un bróker de seguros, adquirí conocimientos y experiencia, que me ayudó a independizarme y emprender. Ahora me capacito 
constantemente para certificarme como bróker de seguros" y la Emprendedora 09 indicó: "Estudié administración y trabajé en el rubro textil hasta que decidi emprender. Me capacité, hice pasantías $y$ viajé a China para conocer sobre telas. Para mantenernos en este mercado competitivo fue necesaria la capacitación y el conocimiento en tecnología”.

Se ha podido observar que las emprendedoras, que luego de analizar sus capacidades, han identificado la necesidad de capacitarse y adaptarse a los nuevos conocimientos lo cual las motiva al desarrollo de sus emprendimientos.

\section{Conclusiones}

La dificultad para emprender de las mujeres del Bicentenario está relacionada con limitaciones en el financiamiento, su rol social y el estereotipo los cuales van cambiando lentamente y donde se reconoce que desde el hogar se debe inculcar la igualdad de género. El temor por la incertidumbre al momento de emprender está asociado principalmente por la posibilidad de descuidar la atención de su hogar por invertir tiempo y dedicación al emprendimiento. En ese sentido busca delegar con terceros la labor familiar para seguir emprendiendo. En la mayoría de los casos, la mujer emprendedora es perseverante ya que debe atender las necesidades económicas de su hogar ocasionadas por la pandemia.

Así mismo, se determinó que las emprendedoras no realizaron una adecuada identificación de oportunidades mediante un pleno análisis del entorno y la planificación no es una práctica cotidiana, por lo que se debería fortalecer la cultura y las capacidades de planificación para un mejor análisis de las oportunidades y prepararse para enfrentar las amenazas en el mediano y largo plazo.

Finalmente, se estableció que las emprendedoras han sabido adaptarse a la tecnología haciendo uso de la misma para poder dar a conocer sus emprendimientos así también han puesto en práctica sus habilidades creativas, sus conocimientos innovados en sus productos y servicios, específicamente en los cambios adicionados para marcar la diferencia con la competencia. La utilización de habilidades desarrolladas en su tiempo libre se convirtió en muchos casos en oportunidades que han sabido aprovechar.

\section{Referencias:}

Alcazar, R., \& Villasana, M. (2015). Construcción y validación de un instrumento para medir competencias emprendedoras. Emprendimiento, creación e incubación de empresas. Durango: EGADE Business School, Tecnológico de Monterrey.

Alene, E. (2020). Determinants that influence the performance of women entrepreneurs in micro and small enterprises in Ethiopia. Journal of Innovation and Entrepreneurship, 9(1). 10.1186/s13731-020-00132-6.

Banco Mundial. (08 de Julio de 2017). Iniciativa de Financiamiento para mujeres emprendedoras. https://www.bancomundial. org/es/programs/women-entrepreneurs/brief/ women-entrepreneurs.

Barbagelata, V. (2019). Estudio de la percepción de mujeres emprendedoras de los factores incidentes en el emprendimiento femenino $y$ el apoyo de entidades externas en Chile. Universidad Técnica Federico Santa María, Santiago.

Beriso, B. S. (2021). Determinants of economic achievement for women entrepreneurs in Ethiopia. Journal of Innovation and Entrepreneurship, 10(1). 10.1186/s13731$\underline{020-00141-5}$

Cámara de Comercio de Cali. (2021). Mujer y Empresa. Contexto, cifras y recomendaciones. Cali.

Chávez, M., Chávez, M., Fuentes, \& Ruiz, J. (2021). Challenging the context:mumpreneurship, copreneurship and sustainable thinking in the entrepreneurial process of women - a case study in Ecuador. Academia Revista Latinoamericana de Administración, 34(3), 368-398. 10.1108/ARLA-07-2020-0172

Cicchiello, A., Kazemikhasragh, A., \& Monferra, S. (2021). In women, we trust! Exploring the sea change in investors' perceptions in equity crowdfunding. Gender in Management, 36(8), 930-951. 10.1108/GM-10-2020-0309

DATUM. (2020). Emprendedores en contexto COVID-19. Lima. 
Delgado, S., Carrasco, R., Chabusa, J., \& Macay, C. (2020). Revista Venezolana de Gerencia, 25(91), 1221-1233.

Ebabu Engidaw, A. (2021). Exploring entrepreneurial culture and its socio-cultural determinants: in case of Woldia University graduating students. Journal of Innovation and Entrepreneurship, 10(1). 10.1186/ s13731-021-00155-7

Encinas, L. M., \& López, G. (2021). Emprendedurismo Femenino: Un estudio multi-caso de factores que influyen en la Intención Emprendedora. Ciencia Latina Revista Multidisciplinar, 5(2), 1642-1659. https://ciencialatina.org/index.php/cienciala/ article/view/374

Fuster, D. (enero-abril de 2019). Investigación cualitativa: Método fenomenológico hermenéutico. Revista Propósitos y Representaciones, 7(1), 201-229.

Galindo, M., Castaño, M., \& Méndez, M. (2021). Effects of the pandemic crisis on entrepreneurship and sustainable development. Journal of Business Research, 137, 345-353. 10.1016/j.jbusres.2021.08.053

Global Reporting Initiative. (2021). Buenas prácticas de sostenibilidad en la Mipyme peruana. Lima: Fondo Cooperación Suiza SECO.

Guananga, L., Rodríguez, M., \& Germán, G. (2018). Situación y gestión del emprendimiento. Quito: Editorial Creative.

Hernández, R., \& Mendoza,C.(2018). Metodología de la investigación. Ciudad de México: McGraw-Hill Interamericana Editores SA.

Huamán, F. A. (2018). La gestión de redes en el fortalecimiento de emprendimientos femeninos: estudio de caso de la Sociedad Picantería Arequipa. Pontificia Universidad Católica del Perú, Tesis de bachiller, Lima. Obtenido de http://hdl.handle. $\underline{\text { net/20.500.12404/11627 }}$

Lin, Y., Fan, D., Shi, X., \& Fu, M. (2021). The effects of supply chain diversification during the COVID-19 crisis: Evidence from Chinese manufacturer. Transportation Research Part E: Logistics and Transportation Review, 155. $\underline{10.1016 / j . t r e .2021 .102493}$

Macedo, E., \& González, E. L. (diciembre de 2017). Emprendimiento y liderazgo de mujeres. Jóvenes en la ciencia, 3(2), 11591163.

Marín, D. (junio de 2020). Oportunidad de emprendedurismo en tiempos de pandemia. Diario Campus, pág. 10.

Medina, A. (2013). Hoja de ruta para emprendedores Crear un negocio en tiempos dificiles. Pirámide.

Mele, C., Russo, T., Tregua, M., \& Amitrano, C. (2021). The millennial customer journey: a Phygital mapping of emotional, behavioural, and social experiences. Journal of Consumer Marketing. 10.1108/JCM-03-2020-3701

Molina,M., Tejeiro, M.,\& Rubio, M. (2021) Never too late to learn: How education helps female entrepreneurs at overcoming barriers in the digital economy. Journal Sustainability (Switzerland). 10.3390/su131911037.

Morrison, A. (2021). La participación laboral de las mujeres en el Perú: 10 mensajes clave y 6 recomendaciones de política. Lima: Fondo Banco Interamericano de Desarrollo.

Ordoñez, D., Castillo, A., \& Rodríguez, I. (2021). Empoderamiento de la mujer en el emprendimiento y la innovación. Revista Población y Desarrollo, 27(52), 69-91.

Querejazu, C. (2020). Aproximación teórica a las causas del emprendimiento. Revista Economía Teórica y Práctica, 28(52), 69-98.

RM 159-2021.MIMP, Orden emérito a las mujeres del bicentenario (Ministerio de la mujer y poblaciones vulnerables 11 de junio de 2021).

Roth, E., Carmona, C., Velasco, C., \& Rodríguez, A. (2011). Algunas dimensiones cognitivas del emprendimiento económico femenino: el papel de los estilos cognitivos en el reconocimiento de oportunidades. Actas del I Congreso Internacional sobre Migraciones en Andalucía Granada, 67-77. 
Ruedas, J. \& Ruiz, R. (2019). Analysis of female entrepreneurship in Latin America. Proceedings of the LACCEI international Multi-conference for Engineering, Education and Technology. 10.18687/ LACCEI2019.1.1.7

Saavedra, M., \& Camarena, M. (2020). Las PYMEs lideradas por mujeres y la capacitación: un estudio exploratorio. SBIR Small Business International Review, 4(2), 1-17.

Sabater, C. (2018). La mujer emprendedora: identidad profesional y factores culturales de género. Revista Femeris, 3(2), 55-78.

Santander, P. (2019). Mujeres emprendedoras: Abordaje desde la Teoría de la Identidad Performativa. Revista Estudos Feministas, Florianópolis, 27(3), e54270. 10.1590/1806$\underline{9584-2019 v 27 \mathrm{n} 354270}$

Serida, J., Alzamora, J., Guerrero, C., Borda, A., \& Morales, O. (2018). Global Entrepreneurship Monitor Perú 2017-2018. Lima: Fondo editorial ESAN.

Sopow, E. (2020), Alinear el bienestar en el lugar de trabajo con el cambio global: un modelo integrado. Journal of Organizational Change Management. 33(5), págs. 909-923. 10.1108/ JOCM-11-2019-0334

Sun, X., Foscht, T., \& Eisingerich, A. B. (2021). Does educating customers create positive word of mouth? Journal of Retailing and Consumer Services, 62(June), 102638. 10.1016/i.jretconser.2021.102638

Surya, A., \& Ati, C. (2022). Do demographic variables make a difference in entrepreneurial leadership style? Case study amongst micro and small in creative economy entrepreneurs in Jakarta, Indonesia. International Journal of Asian Business and Information Management. 12(2). 10.4018/IJABIM.20220701.0a10
Tejeiro, M., Molina, M., \& García, J. (2021). Emprendimiento digital femenino para el desarrollo social y económico: características y barreras en España. REVESCO. Revista de Estudios Cooperativos, 138, e75561. $\underline{10.5209 / \text { reve. } 75561}$

Uzcategui, C., Zambrano, Á., \& Beltrán, A. (2017). Famiemprendimientos, Desafíos de la Mujer Emprendedora. INNOVA Research Journal, 10(10.1), 18-24. 10.33890/innova. v2.n10.1.2017.450

Vásquez, I. (2018). Factores que determinan el emprendimiento femenino caso: Distrito de Chao, al 2018. Universidad César Vallejo, Trujillo. https://hdl.handle. net/20.500.12692/33771

Velásquez, M., \& Portocarrero, W. (2019). Características del Emprendimiento de las Mujeres: caso de mujeres en La Libertad, Perú. Revista Ciencia y Tecnología, 15(1), 89-99. https://revistas.unitru.edu.pe/index. $\mathrm{php} / \mathrm{PGM} /$ article/view/2311

Wong, E., Milaveres, F., Chura, J., Conde, W., \& Aranda, I. (junio de 2021). Asociatividad y gestión de marca: necesidad para ingresar a nuevos mercados - Proyecto Haku Wiñay Tacna. Neumann Business Review, 7(1), 4060.

Yoopetch, C. (2021). Empoderamiento de las mujeres, actitud hacia la toma de riesgos y la intención empresarial en la industria hotelera. Revista Internacional de Investigación en Cultura, Turismo y Hostelería, vol. 15, núm. 1, págs. 59-76. 10.1108/IJCTHR-01-2020-0016

Zegarra, H., Chabusa, J., Legarda, C., \& Espinoza, E. (mayo de 2020). Perfil de la Mujer Emprendedora en Latinoamérica: un marco referencial para Ecuador. Revista Espacios, 41(19), 343-354. 